\title{
On Behaviors of the Energy of Solutions for Some Damped Nonlinear Hyperbolic Equations with $p$-Laplacian
}

\section{Soufiane Mokeddem}

\author{
Laboratory of Biomathematics, Djillali Liabes University, P. B. 89, Sidi Bel Abbes 22000, Algeria \\ s_mokeddem@yahoo.com
}

Keywords: Hyperbolic equations with $p$-Laplace, Decay estimate of energy.

\begin{abstract}
In this paper we are concerned with nonlinear damped hyperbolic equation with p-Laplace of the form $u_{t t}-\Delta_{p} u+\sigma(t)\left(u_{t}-\Delta u_{t}\right)+\omega|u|^{m-2} u=|u|^{r-2} u$. Used the multiplier techniques combined with a nonlinear integral inequalities given by Martinez we established a decay rate estimate for the energy.
\end{abstract}

\section{Introduction}

This paper deals with the decay rate estimate for the energy of the problem

$$
(P)\left\{\begin{array}{l}
u_{t t}-\Delta_{p} u+\sigma(t)\left(u_{t}-\Delta u_{t}\right)+\omega|u|^{m-2} u=|u|^{r-2} u \text { in } \Omega \times[0,+\infty[, \\
u(x, t)=0 \text { on } \partial \Omega \times[0,+\infty[ \\
u(x, 0)=u_{0}(x), u_{t}(x, 0)=u_{1}(x) \text { on } \Omega
\end{array}\right.
$$

where $\Delta_{p} u=\operatorname{div}\left(\left|\nabla_{x} u\right|^{p-2} \nabla_{x} u\right)$ and $p \geq 2$ is real number, $\Omega$ is a bounded domain in $\mathbf{R}^{n}$ with smooth boundary $\partial \Omega$ and the real numbers $\omega, m$ and $r$ satisfy appropriate conditions to be made precise in the sequel.

Several authors have studied the global existence and asymptotic behavior of solutions related to the problem $(P)$ (see for instance [2], [4], [5], [10], [19] and [20]. In all this above cited papers the damping term played an important role in order to give energy decay estimates.

In the case where $\sigma \equiv 1$ with considering $\left(-\Delta^{\alpha}\right) u_{t}$ instead of damping term $\sigma(t)\left(u_{t}-\Delta u_{t}\right)$ in the probleme $(P)$, Gao and $\mathrm{Ma}$ [5] obtained global existence results by means of the Faedo-Galerkin approximations. Further they shown the asymptotic behavior of solutions through the use the integral inequality given by Nakao [11]. However, it will be difficult to proceed by this method with more general functions $\sigma$.

Also, Chen, Yao and Shao [4] investigated the global existence and uniqueness of a solution to an initial boundary problem $u_{t t}-\Delta_{p} u-\Delta u_{t}+g(x, u)=f(x)$. There they established a polynomial decay of energy under certain assumptions on $g$ where $2 \leq p<n$. See also Ye [14, 15], Ma and Soriano [7] for related results.

It is worth mentioning some other papers in connection with asymptotic behavior of solutions to the nonlinear hyperbolic equation with dissipative effects, e.g., [1], [3], [9], [13], [16] and the references therein.

Inspired by [4], we investigated in this paper the decay rate estimate for the energy of the global solutions to the problem $(P)$. For our purpose, we use the multiplier method combined with a nonlinear integral inequalities given by Martinez [8] which depends on the construction of a special weight function that depends on the behavior of $\sigma$.

The paper is organized as follows. In the next section, we present some notations and material needed for our work. The statement and the proof of our main result will be given in section 3 .

To simplify notation, we often write $u(t)$ instead $u(x, t)$ and $u_{t}(t)$ instead $u_{t}(x, t)$. The norm in Lebesgue space $L^{p}(\Omega)$ is denoted by $\|\cdot\|_{p}$, in particular $\|\cdot\|_{2}$ denotes $L^{2}(\Omega)$. We also write equivalent norm $\|\nabla \cdot\|_{p}$ instead of $W_{0}^{1, p}(\Omega)$ norm $\|\cdot\|_{W_{0}^{1, p}(\Omega)}$ and throughout this paper the functions considered are all real valued. 


\section{Preliminaries}

First, suppose that $\sigma: \mathbb{R}_{+} \rightarrow \mathbb{R}_{+}$is a non increasing positive function of class $C^{1}$ on $\mathbb{R}_{+}$, satisfying

$$
\int_{0}^{+\infty} \sigma(\tau) d \tau=+\infty
$$

We denote the total energy functional associated to the solutions of the problem $(P)$ by

$$
E(t)=\frac{1}{2}\left\|u_{t}\right\|_{2}^{2}+\frac{1}{p}\|\nabla u\|_{p}^{p}+\int_{\Omega} F(x, u) d x,
$$

for $u \in W_{0}^{1, p}(\Omega), t \geq 0$ and $F(x, u)=\int_{0}^{u} f(x, s) d s$ and $f(x, u)=\left(\omega|u|^{m-2}-|u|^{r-2}\right) u$.

Before stating our main result, we briefly recall the following result on the existence of a solution of the problem $(P)$.

Theorem 1. Assume that $\left(u_{0}, u_{1}\right) \in W_{0}^{1, p}(\Omega) \times L^{2}(\Omega)$, then the problem $(P)$ admits a solution $u(t)$ in the class

$$
u \in C\left([0, \infty) ; W_{0}^{1, p}(\Omega)\right) \cap C^{1}\left([0, \infty) ; L^{2}(\Omega)\right) .
$$

This result can be established by using Faedo-Galerkin method. The proof closely follows the argument presented in [4], [6] and [13].

We now present some useful lemmas which will be used later.

Lemma 2. Let $u(x, t)$ be a global solution to the problem $(P)$ on $[0, \infty)$. Then we have,

$$
\frac{d}{d t} E(t)=-\sigma(t)\left(\left\|u_{t}\right\|_{2}^{2}+\left\|\nabla u_{t}\right\|_{2}^{2}\right) \leq 0
$$

for all $t \in[0, \infty)$.

This Lemma can be easily proved by multiplying the both sides of the first equation of $(P)$ by $u_{t}$, integrating over $\Omega$ and then using integration by parts.

Lemma 3 Sobolev-Poincaré inequality. Let $p \in \mathbb{N}^{*}$ and $r \in \mathbb{R}$ with $2 \leq r<+\infty(n \leq p)$ or $2 \leq r \leq \frac{n p}{n-p}(n \geq p+1)$. Then there is a constant $c_{*}=c_{*}(\Omega, p, r)$ such that

$$
\|u\|_{r} \leq c_{*}\|\nabla u\|_{p} \quad \text { for } \quad u \in W_{0}^{1, p}(\Omega) .
$$

The case $p=r=2$ gives the known Poincaré's inequality.

In order to solve the energy decay of the problem $(P)$, we use the following lemma.

Lemma 4 [8]. Let $E: \mathbb{R}_{+} \rightarrow \mathbb{R}_{+}$be a non increasing function and $\phi: \mathbb{R}_{+} \rightarrow \mathbb{R}_{+}$an increasing $C^{2}$ function such that

$$
\phi(0)=0 \text { and } \phi(t) \rightarrow+\infty \text { as } t \rightarrow+\infty .
$$

Assume that there exist $q \geq 0$ and $\gamma>0$ such that

$$
\int_{S}^{+\infty} E(t)^{q+1} \phi^{\prime}(t) d t \leq \frac{1}{\gamma} E(0)^{q} E(S), \quad 0 \leq S<+\infty .
$$

Then we have

$$
\begin{aligned}
& \text { if } q=0, E(t) \leq E(0) e^{1-\gamma \phi(t)}, \forall t \geq 0 \\
& \text { if } q>0, \quad E(t) \leq E(0)\left(\frac{1+q}{1+q \gamma \phi(t)}\right)^{\frac{1}{q}}, \forall t \geq 0 .
\end{aligned}
$$




\section{Main results and proof}

We are now ready to state and prove our main result.

Theorem 5. Let $\left(u_{0}, u_{1}\right) \in W_{0}^{1, p}(\Omega) \times L^{2}(\Omega)$ and $n>p>2$. Suppose that (1) holds. Assume further that $p \leq r \leq 2 p$ and $r<m<\frac{n p}{n-p}$. Then there exists a positive constant $c(E(0))$ depending continuously on $E(0)$ such that the solution $u(x, t)$ of the problem $(P)$ satisfies the following energy decay estimate

$$
E(t) \leq\left(\frac{c(E(0))}{\int_{0}^{t} \sigma(\tau) d \tau}\right)^{\frac{p}{p-2}} \quad \forall t>0 .
$$

Proof. Multiplying by $E^{q} \phi^{\prime}(t) u$ on both sides of the first equation of $(P)$ and integrating over $\Omega \times$ $[T, S]$, we obtain that

$$
0=\int_{S}^{T} E^{q} \phi^{\prime} \int_{\Omega} u\left[u_{t t}-\Delta_{p} u+\sigma(t)\left(u_{t}-\Delta u_{t}\right)+f(x, u)\right] d x d t,
$$

where $0 \leq S \leq T \leq+\infty$ and $\phi$ is a function satisfying all the hypotheses of Lemma 4 .

By an integration by parts we see that

$$
\begin{aligned}
0= & {\left[E^{q} \phi^{\prime} \int_{\Omega} u u_{t}\right]_{S}^{T}-\int_{S}^{T}\left(q E^{\prime} E^{q-1} \phi^{\prime}+E^{q} \phi^{\prime \prime}\right) \int_{\Omega} u u_{t} d x d t } \\
& -\int_{S}^{T} E^{q} \phi^{\prime} \int_{\Omega}\left|u_{t}\right|^{2} d x d t+\int_{S}^{T} E^{q} \phi^{\prime} \int_{\Omega}|\nabla u|^{p} d x d t \\
& +\int_{S}^{T} E^{q} \phi^{\prime} \sigma(t) \int_{\Omega} u\left(u_{t}-\Delta u_{t}\right) d x d t+\int_{S}^{T} E^{q} \phi^{\prime} f(x, u) d x d t .
\end{aligned}
$$

Hence from the definition of energy and a simple computation we get

$$
\begin{aligned}
& p \int_{S}^{T} E^{q+1} \phi^{\prime} d t=-\left[E^{q} \phi^{\prime} \int_{\Omega} u u_{t}\right]_{S}^{T}+\int_{S}^{T}\left(q E^{\prime} E^{q-1} \phi^{\prime}+E^{q} \phi^{\prime \prime}\right) \int_{\Omega} u u_{t} d x d t \\
& \quad+\left(\frac{p}{2}+1\right) \int_{S}^{T} E^{q} \phi^{\prime} \int_{\Omega}\left|u_{t}\right|^{2} d x d t-\int_{S}^{T} E^{q} \phi^{\prime} \sigma(t) \int_{\Omega} u\left(u_{t}-\Delta u_{t}\right) d x d t \\
& \quad+\int_{S}^{T} E^{q} \phi^{\prime} \int_{\Omega}(p F(u)-u f(x, u)) d x d t .
\end{aligned}
$$

We must estimate every terms of right-hand side of (6) to arrive at a similar inequality as (4).

Define,

$$
\phi(t)=\int_{0}^{t} \sigma(\tau) d \tau
$$


so that $\phi$ is a nondecreasing function of class $C^{2}$ on $\mathbb{R}_{+}$and the hypothesis (1) ensures that

$$
\phi(t) \rightarrow+\infty \text { as } t \rightarrow+\infty \text {. }
$$

Exploiting Cauchy-Schwartz inequality, Sobolev-poincaré inequality and the definition of energy we get

$$
\int_{\Omega} u u_{t} d x \leq\|u\|_{2}\left\|u_{t}\right\|_{2} \leq c\|\nabla u\|_{p}\left\|u_{t}\right\|_{2} \leq c E(t)^{\frac{1}{p}} E(t)^{\frac{1}{2}} .
$$

Using nonincreasing property of $E$ and the fact that $\phi^{\prime}$ is a bounded non negative function on $\mathbb{R}_{+}$(we denote by $\mu$ its maximum) we obtain that

$$
-\left|\left[E^{q} \phi^{\prime} \int_{\Omega} u u_{t} d x\right]_{S}^{T}\right| \leq c \mu E(S)^{q+\frac{1}{2}+\frac{1}{p}}
$$

here and from now on, $c$ denotes a positive constant which can be different from line to line.

Similarly we have

$$
\begin{aligned}
& \left|\int_{S}^{T}\left(q E^{\prime} E^{q-1} \phi^{\prime}+E^{q} \phi^{\prime \prime}\right) \int_{\Omega} u u_{t} d x d t\right| \\
& \quad \leq c \mu \int_{S}^{T}-E^{\prime} E^{q-\frac{1}{2}+\frac{1}{p}} d t+\int_{S}^{T} c E^{q+\frac{1}{2}+\frac{1}{p}}\left(-\phi^{\prime \prime}\right) d t \leq c \mu E(S)^{q+\frac{1}{2}+\frac{1}{p}} .
\end{aligned}
$$

On the other hand, from Lemma 2 we have that

$$
\begin{aligned}
(1+ & \left.\frac{p}{2}\right) \int_{S}^{T} E^{q} \phi^{\prime} \int_{\Omega}\left|u_{t}\right|^{2} d x d t \leq\left(1+\frac{p}{2}\right) \int_{S}^{T} E^{q} \phi^{\prime} \int_{\Omega}\left(\left|u_{t}\right|^{2}+\left|\nabla u_{t}\right|^{2}\right) d x d t \\
& \leq\left(1+\frac{p}{2}\right) \int_{S}^{T} E^{q} \phi^{\prime}\left(-\frac{E^{\prime}(t)}{\sigma(t)}\right) d t \leq c E^{q+1}(S) .
\end{aligned}
$$

We also need to estimate

$$
\int_{S}^{T} E^{q}(t) \phi^{\prime} \int_{\Omega}(p F(u)-u f(u)) d x d t .
$$

From Sobolev-Poincaré inequality, there exists $r^{\prime}>0$ such that

$$
r^{\prime}\|u\|_{p}^{p} \leq\|\nabla u\|_{p}^{p} \quad \forall u \in W_{0}^{1, p}(\Omega) .
$$

As $f(x, u)=\left(\omega|u|^{m-2}-|u|^{r-2}\right) u$, with $F(x, u)=\int_{0}^{u} f(x, s) d s$ we have,

$$
F(u)=\omega \frac{|u|^{m}}{m}-\frac{|u|^{r}}{r} .
$$

We also notice that there exists $\omega^{\prime}>0$ where $\omega>\omega^{\prime}$ so that,

$$
\frac{\omega}{2 m}|u|^{m} \leq \frac{\omega^{\prime}}{2 p}|u|^{p}+F(u) \quad \forall u \in \mathbf{R} .
$$


Thus, we obtain from (11) that

$$
\begin{aligned}
\int_{S}^{T} E^{q}(t) & \phi^{\prime} \int_{\Omega}(p F(u)-u f(x, u)) d x d t=\int_{S}^{T} E^{q}(t) \phi^{\prime} \int_{\Omega}\left(\frac{r-p}{r}|u|^{r}-\frac{r(m-p)}{m}|u|^{m}\right) d x d t \\
& \leq \int_{S}^{T} E^{q}(t) \phi^{\prime} \int_{\Omega} \frac{r-p}{r}|u|^{r} d x d t=\int_{S}^{T} E^{q}(t) \phi^{\prime} \int_{\Omega}(r-p)\left(\frac{\omega}{m}|u|^{m}-F(u)\right) d x d t,
\end{aligned}
$$

and taking into account (12), we have

$$
\begin{aligned}
\int_{S}^{T} E^{q}(t) & \phi^{\prime} \int_{\Omega}(p F(u)-u f(x, u)) d x d t \\
& \leq \int_{S}^{T} E^{q}(t) \phi^{\prime} \int_{\Omega}(r-p)\left(\frac{\omega^{\prime}}{p}|u|^{p}+2 F(u)-F(u)\right) d x d t \\
& \leq \int_{S}^{T} E^{q}(t) \phi^{\prime} \int_{\Omega}(r-p)\left(\frac{\omega^{\prime}}{p}|u|^{p}+F(u)\right) d x d t
\end{aligned}
$$

Using the definition of the energy $E(t)$ we see that

$$
\int_{\Omega}\left(\frac{\omega^{\prime}}{p}|u|^{p}+F(u)\right) d x \leq c E(t)
$$

Consequently,

$$
\int_{S}^{T} E^{q}(t) \phi^{\prime} \int_{\Omega}(p F(u)-u f(u)) d x d t \leq c(r-p) \int_{S}^{T} E^{q} E(t) \phi^{\prime} d t \leq c(r-p) \int_{S}^{T} E^{q+1}(t) \phi^{\prime} d t .
$$

The remaining term of the right hand side of (6) can be estimate as follows,

$$
\begin{aligned}
& \int_{S}^{T} E^{q} \phi^{\prime} \sigma(t) \int_{\Omega} u\left(u_{t}-\Delta u_{t}\right) d x d t \\
& =\int_{S}^{T} E^{q} \phi^{\prime} \sigma(t) \int_{\Omega} u u_{t} d x d t-\int_{S}^{T} E^{q} \phi^{\prime} \sigma(t) \int_{\Omega} u \Delta u_{t} d x d t \\
& =\int_{S}^{T} E^{q} \phi^{\prime} \sigma(t) \int_{\Omega} u u_{t} d x d t+\int_{S}^{T} E^{q} \phi^{\prime} \sigma(t) \int_{\Omega} \nabla u \nabla u_{t} d x d t \\
& \leq \int_{S}^{T} E^{q} \phi^{\prime} \sigma(t) \int_{\Omega} \nabla u \nabla u_{t} d x d t
\end{aligned}
$$

We received from Hölder inequality and Sobolev-Poincaré inequality that

$$
\left|\int_{S}^{T} E^{q} \phi^{\prime} \sigma(t) \int_{\Omega} \nabla u \nabla u_{t} d x d t\right| \leq \int_{S}^{T} E^{q} \phi^{\prime} \sigma(t)\|\nabla u\|_{p}\left\|\nabla u_{t}\right\|_{\frac{p}{p-1}} d t
$$


We also have

$$
\left\|\nabla u_{t}\right\|_{\frac{p}{p-1}} \leq c|\Omega|^{\frac{p-2}{2 p}}\left\|\nabla u_{t}\right\|_{2} \leq c|\Omega|^{\frac{p-2}{2 p}}\left(\frac{-E^{\prime}(t)}{\sigma(t)}\right)^{\frac{1}{2}}
$$

This gives

$$
\begin{gathered}
\left|\int_{S}^{T} E^{q} \phi^{\prime} \sigma(t) \int_{\Omega} \nabla u \nabla u_{t} d x d t\right| \leq c \int_{S}^{T} E^{q+\frac{1}{p}} \phi^{\prime} \sigma(t)\left(\frac{-E^{\prime}(t)}{\sigma(t)}\right)^{\frac{1}{2}} d t \\
=c \int_{S}^{T} E^{q+\frac{1}{p}} \phi^{\prime} \sigma(t)^{\frac{1}{2}}\left(-E^{\prime}(t)\right)^{\frac{1}{2}} d t .
\end{gathered}
$$

Further, by Young inequality, we have for $\varepsilon>0$

$$
\begin{gathered}
\left|\int_{S}^{T} E^{q} \phi^{\prime} \sigma(t) \int_{\Omega} \nabla u \nabla u_{t} d x d t\right| \leq \frac{c \varepsilon^{2}}{2} \int_{S}^{T} E^{2\left(q+\frac{1}{p}\right)}\left(\phi^{\prime}(t)\right)^{2} \sigma(t) d t+\frac{c}{2 \varepsilon^{2}} E(S) \\
=\frac{c \varepsilon^{2}}{2} \int_{S}^{T} E^{2\left(q+\frac{1}{p}\right)} \phi^{\prime}(t)(\sigma(t))^{2} d t+\frac{c}{2 \varepsilon^{2}} E(S) .
\end{gathered}
$$

Thus takes, $2\left(q+\frac{1}{p}\right)=q+1$, so that $q=(p-2) / p$.

Then substituting the estimates (8), (9), (10), (14) and (13) into (6), we get

$$
\begin{gathered}
\int_{S}^{T} E^{q+1} \phi^{\prime}(t) d t \leq c E(S)^{q+\frac{1}{2}+\frac{1}{p}}+c^{\prime} E(S)^{q+1}+c^{\prime \prime} E(S) \\
\leq\left(\frac{c E(0)^{q-\frac{1}{2}+\frac{1}{p}}+c^{\prime} E(0)^{q}+c^{\prime \prime}}{E(0)^{q}}\right) E(0)^{q} E(S),
\end{gathered}
$$

where $c, c^{\prime}$ and $c^{\prime \prime}$ are different positive constants independent of $E(0)$.

Let $T \rightarrow+\infty$, we have from (15) that

$$
\int_{S}^{+\infty} E^{q+1} \phi^{\prime}(t) d t \leq\left(\frac{c E(0)^{q-\frac{1}{2}+\frac{1}{p}}+c^{\prime} E(0)^{q}+c^{\prime \prime}}{E(0)^{q}}\right) E(0)^{q} E(S), \quad \forall S \geq 0,
$$

Thus we receive from Lemma 4 that

$E(t) \leq\left(c E(0)^{q-\frac{1}{2}+\frac{1}{p}}+c^{\prime} E(0)^{q}+c^{\prime \prime}\right)^{\frac{1}{q}}\left(\frac{1+q}{q}\right)^{\frac{1}{q}}\left(\int_{0}^{t} \sigma(s) d s\right)^{-\frac{1}{q}}=(c(E(0)))^{\frac{1}{q}}\left(\int_{0}^{t} \sigma(s) d s\right)^{-\frac{1}{q}}$,

where $c(E(0))=\left(c E(0)^{q-\frac{1}{2}+\frac{1}{p}}+c^{\prime} E(0)^{q}+c^{\prime \prime}\right)\left(\frac{1+q}{q}\right)$ is a positive constant depending on $E(0)$. As $q=(p-2) / p$, we have

$$
E(t) \leq(c(E(0)))^{\frac{p}{p-2}}\left(\int_{0}^{t} \sigma(s) d s\right)^{-\frac{p}{p-2}}, \quad t \in[0,+\infty)
$$

The proof is thus finished. 


\section{References}

[1] N. Amroun, S. Mimouni, Asymptotic behaviour of solutions for some weakly dissipative wave equations of p-Laplacian type, Applied Mathematics E-Notes. 11 (2011) 175-183.

[2] A. Benaissa, S. A. Messaoudi, Exponential decay of solutions of a nonlinearly damped wave equation, Nonlinear Differ. Equ. Appl. 12(4) (2005) 391-399.

[3] A. Benaissa, S. Mokeddem, Decay estimates for the wave equation of p-Laplacian type with dissipation of m-Laplacian type, Math. Methods Appl. Sci. 30(2) (2007) 237-247.

[4] C. Chen, H. Yao, L. Shao, Global Existence, Uniqueness, and Asymptotic behavior of solution for p-Laplacian Type wave equation. J. Inequal. Appl. 2010, Art. ID 216760.

[5] H. Gao, T. F. Ma, Global solutions for a nonlinear wave equation with the p-Laplacian operator, Electronic Journal of Qualitative Theory of Differential Equations. 11 (1999) 1-13.

[6] J. L. Lions, Quelques Méthodes de Résolution des Problèmes aux Limites non Linéaires, DunodGauthier Villars, Paris, France, 1969.

[7] T. F. Ma, J. A. Soriano, On weak solutions for an evolution equation with exponential nonlinearities, Nonlinear Anal. 37(8) (1999) 1029-1038.

[8] P. Martinez, A new method to decay rate estimates for dissipative systems, ESAIM Control Optim. Calc. Var. 4 (1999) 419-444.

[9] S. Mokeddem, Kh. B. W. Mansour, Asymptotic behaviour of solutions for $p$-Laplacian wave equation with $m$-Laplacian dissipation, Z. Anal. Anwend. 33(3) (2014) 259-269.

[10] M. I. Mustafa, A. S. Messaoudi, General energy decay rates for a weakly damped wave equation, Commun. Math. Anal. 9(2) (2010) 67-76.

[11] M. Nakao, A difference inequality and its applications to nonlinear evolution equations, Journal of the Mathematical Society of Japan. 30(4) (1978) 747-762.

[12] D. H. Sattinger, On global solution of nonlinear hyperbolic equations, Arch. Ration. Mech. Anal. 30 (1968) 148-172.

[13] Z. Yang, Existence and asymptotic behaviour of solutions for a class of quasilinear evolution equations with nonlinear damping and source terms, Mathematical Methods in the Applied Sciences. 25(10) (2002) 795-814.

[14] Y. Ye, Existence of global solutions for some nonlinear hyperbolic equation with a nonlinear dissipative term, Journal of Zhengzhou University. 29(3) (1997) 18-23.

[15] Y. Ye, On the decay of solutions for some nonlinear dissipative hyperbolic equations, Acta Mathematicae Applicatae Sinica. English Series. 20(1) (2004) 93-100.

[16] Y. Ye, Exponential decay of energy for some nonlinear hyperbolic equations with strong dissipation, Adv. Difference Equ. (2010) Article ID 357404. 
[17] Y. Ye, Global existence and asymptotic behavior of solutions for some nonlinear hyperbolic equation, J. Inequal. Appl. (2010) Article ID 895121.

[18] E. Zuazua, Exponential decay for the semilinear wave equation with locally dis-tributed damping, Comm. Partial Differential Equations. 15 (1990) 205-235.

[19] E. Zuazua, Uniform Stabilization of the wave equation by nonlinear boundary feed-back, SIAM J. Control Optim. 28(2) (1990) 466-477. 